\title{
Treatment of Dry Eye Disease with Autologous Platelet-Rich Plasma: A Prospective, Interventional, Non-Randomized Study
}

\author{
Jorge L. Alio - Alejandra E. Rodriguez - Renan Ferreira-Oliveira • \\ Dominika Wróbel-Dudzińska · Ahmed A. Abdelghany
}

Received: June 21, 2017 / Published online: August 8, 2017

(C) The Author(s) 2017. This article is an open access publication

\section{ABSTRACT}

Introduction: The objective of this study was to evaluate the use of autologous platelet-rich plasma (PRP) eye drops as monotherapy for the treatment of moderate to severe cases of dry eye disease.

Methods: Three hundred and sixty-eight patients with moderate to severe dry eye disease (DED) were included in this prospective case series. Subjects were classified as evaporative DED (EDED) or aqueous deficient DED (ADDED). Improvement of the DED subjective symptoms, corneal fluorescein staining (CFS),

Enhanced content To view enhanced content for this article go to http://www.medengine.com/Redeem/ 58F8F0600F18A0AF.

J. L. Alio $(\bowtie) \cdot$ R. Ferreira-Oliveira .

D. Wróbel-Dudzińska · A. A. Abdelghany

Department of Cornea and Refractive Surgery,

VISSUM, Alicante, Spain

e-mail: jlalio@vissum.com

J. L. Alio - A. A. Abdelghany

Division of Ophthalmology, Universidad Miguel

Hernández, Alicante, Spain

A. E. Rodriguez - R. Ferreira-Oliveira .

D. Wróbel-Dudzińska

Research and Development Department, VISSUM, Alicante, Spain

A. A. Abdelghany

Ophthalmology Department, Faculty of Medicine,

Minia University, Minia, Egypt and corrected distance visual acuity (BCVA) were evaluated. We also analysed how many rounds of PRP therapy were used.

Results: Two hundred and ninety-seven (80.7\%) patients were women, and $71(19.3 \%)$ were men. Two hundred and thirty-two (63\%) patients had EDED, while 136 (37\%) had ADDED. After 6 weeks of monotherapy treatment with autologous PRP, dry eye symptoms improved in 322 (87.5\%) cases. A decrease of CFS was observed in 280 (76.1\%) patients. One hundred and six (28.8\%) patients improved at least 1 line of BCVA. The scores in the ocular Surface Disease Index and the Oxford scale of corneal fluorescein staining decreased statistically after the treatment $(p<0.05)$.

Conclusion: The topical use of autologous platelet-rich plasma as monotherapy is an effective treatment to improve signs and symptoms in patients suffering from moderate to severe chronic DED.

Keywords: Autologous platelet-rich plasma; Autologous serum; Dry eye; Plasma rich in growth factors; PRP

\section{INTRODUCTION}

The definition of dry eye disease (DED), updated in 2007 by the International Dry Eye Workshop, is a multifactorial disease of the ocular surface and tears that produce symptoms of discomfort, visual disturbance, and tear film instability with 
potential damage to the ocular surface. It is accompanied by increased osmolarity of the tear film and inflammation of the ocular surface [1].

The prevalence of dry eye reported in the literature varies from $4 \%$ to $30 \%$ [2-4]. Such wide discrepancies in the data may result from racial differences, different definitions, and the variety of tests used to determine DED. There are two main mechanisms that explain this ocular surface dysfunction: aqueous deficiency and excessive evaporation of the tear film. The aqueous deficient dry eye disease (ADDED) is characterized by an insufficient volume of tears due to dysfunction of the lacrimal glands and obstruction of the lacrimal ducts. This mechanism is also related to autoimmune diseases such as Sjögren's syndrome, lupus, and rheumatoid arthritis. Meibomian gland dysfunction, eyelid problems (infrequent blinking, entropion, ectropion) are typical causes of evaporative dry eye. Poor tear film quality is the result of tear hyperosmolarity and goblet cell mucin deficiency [5].

The main standard treatment for dry eye is topical administration of artificial tears, although the expected results are not optimal and often ineffective. This has led to the use of other therapeutic strategies based on hemoderivatives. Autologous serum (AS) has been suggested as a more adequate treatment for severe DED over preservative-free artificial molecules, with variable success rates $[6,7]$. Platelet rich plasma (PRP) and plasma rich in growth factors (PRGF) have also been reported as successful treatments for moderate to severe dry eye, presenting advantages over AS due to its richer concentration of growth factors, anti-inflammatory cytokines, and other platelet derivatives, which could be beneficial for the required ocular surface restoration in moderate to severe forms of dry eye $[5,8]$.

Autologous PRP is a hemoderivative with a high concentration of platelets obtained through a relatively simple process, which requires minimal manipulation and no addition of any other particular substance [9]. A recent study has shown that these components help in the proliferation, migration, and differentiation of corneal epithelial cells [10]. We have previously reported the outcomes of a pilot investigation with successful use of autologous PRP in the treatment of symptomatic dry eye [5].

The present study is a large non-randomised consecutive series of cases, conducted to evaluate prospectively the efficacy of topical administration of autologous PRP as monotherapy for the treatment of symptoms and clinical signs in cases affected by moderate to severe forms of chronic DED.

\section{METHODS}

Prospective, interventional, non-randomized study conducted at the VISSUM Ophthalmology Institute in Alicante, Spain. Ethical committee approval was obtained for the purpose of this prospective investigation. The study followed the tenets of the Declaration of Helsinki [11], and adequate informed consent was obtained from all patients included in the study.

Adult patients with moderate to severe chronic DED (for 6 months or more)-according to the Dry Eye severity grading scheme proposed by the Dry Eye WorkShop (DEWS) [1] and Baudouin et al. [12]-were included in the study. This scheme takes into account the following parameters: ocular discomfort, visual symptoms, conjunctival injection, conjunctival and corneal fluorescein staining (CFS), corneal/ tear signs, lid/meibomian glands, tear film break-up time (TBUT), and Schirmer test score [1].

Patients with previous ocular refractive surgery (LASIK, LASEK, or PRK), inability to attend the 6th week follow-up visit and patients who did not complete the follow-up protocol were excluded.

Any topical medication used formerly by the patient for the treatment of the DED was stopped $48 \mathrm{~h}$ before starting PRP treatment. Treatment consisted of monotherapy with PRP eye drops used on both eyes, one drop six times per day for at least 6 consecutive weeks (one round). When the improvement was initially poor or not satisfactory enough, some patients needed more than one round of PRP treatment. 
Treatment response was evaluated by the results of the subjective questionnaires, the slit lamp examination and BCVA.

\section{Main Outcome Measures}

Patients were classified as evaporative DED (EDED) or aqueous deficient DED (ADDED) according to the criteria presented by Lemp et al. [13], in which by using the most severe measurement of both eyes, subjects were considered to have pure aqueous-deficient dry eye (ADDED) with Schirmer's test values of $<7 \mathrm{~mm}$ and MGD grades of $\leq 5$, whereas patients with Schirmer's values of $\geq 7 \mathrm{~mm}$ and MGD grades of $>5$ were classified as purely evaporative dry eye [13].

Subjective symptoms were evaluated based on the ocular surface disease index (OSDI) self-administered questionnaire, which was completed once at the time of enrollment in the study (baseline) and again after treatment with E-PRP. OSDI scores range from 0 to 100 according to the severity of the DED symptoms. Patients were also directly asked to report any change in their perception of ocular redness, foreign body sensation, itching, photophobia, dryness, and pain, based on a five-point scale in which $0=$ no change, $1=$ slight improvement, $2=$ moderate improvement, $3=$ very good, and $4=$ excellent.

Best corrected distance visual acuity (BCVA) was measured with Snellen charts on a conventional projection screen with far distance correction and without using any eye drops $1 \mathrm{~h}$ before measurement. Conversion to decimal scale and lines of vision were posteriorly done to compare results.

Corneal fluorescein staining (CFS) was used to evaluate corneal superficial punctate epithelial keratitis. The modified Oxford scale, ranging from 0 (no staining dots) to 5 (worst staining condition), was used for this purpose. A decrease in CFS scores in at least one quadrant to total disappearance from baseline indicated improvement [14].

\section{Autologous PRP Preparation}

Autologous PRP was prepared according to the protocol described by our group in a previous study [9]. The patient's blood was extracted into $10-\mathrm{mL}$ sterile tubes containing $1 \mathrm{~mL}$ sodium citrate acting as an anticoagulant. Centrifugation of total blood at optimal condition was used to achieve enrichment of platelets in plasma fraction. Under a laminar flow cabin, 3-4 mL of platelet-rich plasma was aspirated and put into sterilized amber glass bottles with eye drop applicators. A haemocytometer (Mythic 18, Domenech, Madrid, Spain) was used to quantify the number of platelets in whole blood after the centrifugation in order to obtain the maximum enrichment. Patients were instructed to maintain good personal hygiene: to wash their hands before the application of the product, keep the area of the application clean and not touch the eye dropper. The bottle in use should be kept at $+4-8{ }^{\circ} \mathrm{C}$ for 1 week and the remaining bottles at $-20^{\circ} \mathrm{C}$.

\section{Patient Follow-Up}

An independent observer followed up each case. Data were recorded for the purpose of this report at the initial visit, and at the end of each round of 6 weeks of monotherapy treatment. Follow-up visits included an interview with the patient assessing the presence of ocular symptoms, and ophthalmologic examination to register all the clinical findings. Improvement or failure was assessed according to the criteria described above.

\section{Statistical Analysis}

Shapiro-Wilk test was used to study the normal distribution of the data. When the parameters analysed were quantitative variables (mean and standard deviations) we used paired 2-tailed Student $t$ test or the Wilcoxon matched-pairs test. For qualitative variables descriptive statistics were used. The statistical significance level chosen was $p \leq 0.05$. Correlations were performed using the non-parametric Spearman test. Microsoft Office Excel 2010 and SPSS 13.0 package were used for analysis of data. 
Table 1 Blood cell count before (whole blood) and after centrifugation (PRP)

\begin{tabular}{llll}
\hline & Platelets $\times \mathbf{1 0}^{\mathbf{3}} / \mathbf{\mu L}$ & $\mathbf{R B C} \times \mathbf{1 0 ^ { \mathbf { 6 } } / \boldsymbol { \mu L }}$ & $\mathbf{W B C} \times \mathbf{1 \mathbf { 0 } ^ { 3 } / \boldsymbol { \mu L }}$ \\
\hline Whole blood (Mean \pm SD) & $201.48 \pm 51.39$ & $4.09 \pm 0.45$ & $4.60 \pm 1.60$ \\
PRP (Mean \pm SD) & $340.07 \pm 98.37^{*}$ & $0.02 \pm 0.05$ & $0.84 \pm 0.50$ \\
Concentration index & 1.71 & - & - \\
Reduction \% & - & $-99.5^{*}$ & $-81.2^{*}$ \\
\hline
\end{tabular}

$R B C$ red blood cells, $W B C$ white blood cells, $S D$ standard deviation

${ }^{*} p<0.05$

\section{RESULTS}

THree hundred and sixty-eight patients with DED were included in this study. Seventy-one patients were men (19.3\%), and 297 patients were women (80.7\%) with ages ranging from 18 to 77 years (mean $50.1 \pm 15.8$ ) and 18 to 89 years (mean $56.1 \pm 16.6$ ), respectively.

Before treatment with PRP, 121 patients $(32.9 \%)$ received topical anti-inflammatory therapy for DED without beneficial results. Also, 19 subjects $(5.2 \%)$ had been treated with autologous serum eye drops without noticing any improvement.

Two hundred and thirty-two patients (63\%) had evaporative DED (EDED), while 136 patients (37\%) had aqueous deficient DED (ADDED). Of the ADDED patients, 23 (16.9\%) had Sjögren's syndrome dry eye, while 113 $(83.1 \%)$ had non-Sjögren's ADDED.

Mean values of blood cell count before and after PRP preparation are shown in Table 1 . The platelets count in the PRP samples was significantly higher than baseline value in the whole blood $(p<0.05)$, and the concentration index was 1.71 . Red blood cells and leukocytes in the PRP decreased significantly after preparation, $99.5 \%$ and $81.2 \%$, respectively $(p<0.05)$.

The Schirmer's test value in the evaporative DED patients improved from $9.5 \pm 3.6 \mathrm{~mm}$ to $13.8 \pm 8.7$ after the treatment $(p<0.05)$, and in the ADDED patients from $4.7 \pm 2.7$ to $6.4 \pm 2.4$ $(p<0.05)$.

Regarding subjective symptoms of DED after the treatment with PRP, 322 patients $(87.5 \%)$ reported an improvement with respect to its previous condition, showing reduction or absence of symptoms, while 46 (12.5\%) did not experience any change in symptoms. The OSDI scores previous to the PRP treatment were $42.9 \pm 26.7$ in the EDED patients, and $69.6 \pm 30.6$ in the ADDED patients. Both groups decreased significantly after the PRP treatment to $18.1 \pm 17.0$ and $43.2 \pm 14.5$, respectively. These differences were statistically significant $(p<0.05)$.

Mean decimal BCVA of all subjects improved from $0.75 \pm 0.3$ at baseline to $0.82 \pm 0.2$ after the treatment with PRP. Globally these values were not statistically significant. One hundred and six patients $(28.8 \%)$ experienced an improvement of one or more lines of vision. No improvement in vision was observed in 246 (66.9\%) patients, while $16(4.3 \%)$ patients lost one line of vision. The gain in lines of vision was significantly correlated with the improvement in the CFS score $(p=0.04, r=0.61)$. Outcome results in each group are presented in Table 2.

All cases enrolled in the study initially showed superficial punctate keratopathy, with modified Oxford scale scores ranging from 0.5 to 4 . After the treatment, a decrease in the area with fluorescein staining was observed in 280 (76.1\%) patients. A decrease of at least 1 point in the modified Oxford scale score was indicative of improvement. Seventy-eight (21.2\%) cases did not show any changes, while ten cases $(2.7 \%)$, all women who had received one round of PRP, showed worsening in the extension of punctate keratopathy. The mean CFS score previously to the treatment was $1.43 \pm 0.94$ and after the PRP $0.24 \pm 0.43$ in the EDED patients. In the ADDED patients, the scores were $1.70 \pm 0.90$ and $0.39 \pm 0.67$, before and after, respectively. Both groups decreased significantly after the PRP treatment $(p<0.05)$. 
Table 2 Main outcome measures per DEDs groups after PRP treatment

\begin{tabular}{|c|c|c|c|c|c|c|}
\hline \multirow[t]{2}{*}{ Main outcome measures } & \multirow[t]{2}{*}{ Scales } & \multirow{2}{*}{$\begin{array}{l}\text { DED } \\
n=368 \\
n(\%)\end{array}$} & \multirow{2}{*}{$\begin{array}{l}\text { Evaporative DED } \\
n=232 \\
n(\%)\end{array}$} & \multicolumn{3}{|c|}{ Aqueous deficient DED } \\
\hline & & & & $\begin{array}{l}\mathrm{AD} \\
n=136 \\
n(\%)\end{array}$ & $\begin{array}{l}S S \\
n=23 \\
n(\%)\end{array}$ & $\begin{array}{l}\text { Non-SS } \\
n=113 \\
n(\%)\end{array}$ \\
\hline \multirow[t]{5}{*}{ Rounds of PRP } & 1 & $237(64.4)$ & $161(69.4)$ & $76(55.9)$ & $9(39.1)$ & $67(59.3)$ \\
\hline & 2 & $67(18.2)$ & $41(17.7)$ & $26(19.1)$ & $4(17.4)$ & $22(19.5)$ \\
\hline & 3 & $30(8.2)$ & $11(4.7)$ & $19(14.0)$ & $5(21.8)$ & $14(12.4)$ \\
\hline & 4 & $17(4.6)$ & $10(4.3)$ & $7(5.1)$ & $1(4.3)$ & $6(5.3)$ \\
\hline & 5 & $17(4.6)$ & $9(3.9)$ & $8(5.9)$ & $4(17.4)$ & $4(3.5)$ \\
\hline \multirow{5}{*}{$\begin{array}{l}\text { Improvement in subjective } \\
\text { symptoms }\end{array}$} & 4 & $23(6.3)$ & $17(7.3)$ & $6(4.4)$ & $1(4.3)$ & $5(4.4)$ \\
\hline & 3 & $130(35.3)$ & $73(31.5)$ & $57(41.9)$ & $10(43.6)$ & $47(41.6)$ \\
\hline & 2 & $106(28.8)$ & $72(31.0)$ & $34(25.0)$ & $8(34.7)$ & $26(23.0)$ \\
\hline & 1 & $63(17.1)$ & $41(17.7)$ & $22(16.2)$ & $2(8.7)$ & $20(17.7)$ \\
\hline & 0 & $46(12.5)$ & $29(12.5)$ & $17(12.5)$ & $2(8.7)$ & $15(13.3)$ \\
\hline \multirow[t]{3}{*}{ Gain in lines of vision } & 1 or more & $106(28.8)$ & $52(22.4)$ & $54(39.7)$ & $10(43.5)$ & $44(38.9)$ \\
\hline & No gain & $246(66.9)$ & $173(74.6)$ & $73(53.7)$ & $12(52.2)$ & $61(54.0)$ \\
\hline & Lost & $16(4.3)$ & $7(3.0)$ & $9(6.6)$ & $1(4.3)$ & $8(7.1)$ \\
\hline \multirow[t]{3}{*}{ CFS } & Improvement & $280(76.1)$ & $182(78.4)$ & $98(72.1)$ & $14(60.9)$ & $84(74.4)$ \\
\hline & No change & $78(21.2)$ & $43(18.6)$ & $35(25.7)$ & $9(39.1)$ & $26(23.0)$ \\
\hline & Worsening & $10(2.7)$ & $7(3.0)$ & $3(2.2)$ & $0(0.0)$ & $3(2.6)$ \\
\hline
\end{tabular}

1 round 6 weeks consecutively, $S S$ Sjögren's syndrome, $A D$ aqueous deficient, $C F S$ corneal fluorescein staining

Eight $(2.2 \%)$ patients presented corneal ulcer due to severe dry eye before treatment with PRP. The associated pathologies in these subjects were thyroid eye disease, exposure keratopathy, uncontrolled cicatricial pemphigoid, graft versus host disease (GVHD), corneal leucomas (2), and severe non-Sjögren ADDED (2). They used two to three rounds of treatment with PRP, except for the patient with GVHD who needed up to five rounds. All of them had significant improvement in CFS parameters although they continued complaining of dry eye symptoms. Only one patient $(0.02 \%)$ reported intolerance to the use of PRP eye drops due to extreme discomfort and stinging sensation at the time of instillation.
Regarding the number of rounds of PRP treatment, of the 368 patients treated, 237 $(64.4 \%)$ received only one round of PRP (6 weeks consecutively) and 131 (35.6\%) between two and five rounds. More ADDED patients used three cycles of PRP $(p<0.05)$, while no statistically significant difference was found between EDED and ADDED groups for other numbers of rounds. More patients with EDED (64.9\%) used only one round of PRP in comparison with ADDED (55.9\%), but this difference was not statistically significant. More rounds of PRP produced greater improvement in the subjective symptoms in ADDED $(r=0.64)$ than in $\operatorname{EDED}(r=0.39)$ patients, while in terms of CFS, two or more cycles of PRP 
produced greater improvement in EDED $(r=0.62)$ than in ADDED $(r=0.51)$ patients, although these differences were not statistically significant $(p>0.05)$.

\section{DISCUSSION}

Dry eye disease comprehends a group of entities with an ocular surface disorder that can be caused by several factors and has different degrees of severity. Some patients experience mild symptoms that can be effectively treated with artificial tears, while others develop severe symptoms and complications of keratoconjunctivitis sicca such as infection, epithelial metaplasia, glandular dysfunction, and neurotrophic damage. The most frequently reported complaints of patients with dry eye syndrome are sandy-gritty eye irritation, burning, intermittent sharp pain, and photophobia, but often there is no association between the signs and symptoms of DED [15]. DED can also affect visual acuity due to tear film instability and induction of corneal irregularity.

At present, the first line of therapy for DED are artificial tears, which provide symptomatic relief by maintaining the ocular surface moistened, but do not resolve underlying causes. Other disadvantages include the need for frequent drop application and lower efficacy after long standing treatment. In more severe cases, anti-inflammatory therapy such as topical corticosteroids or cyclosporine $\mathrm{A}$ are used to improve signs and symptoms of DED [16]. An alternative approach in the treatment of moderate to severe dry eye disease is the use of autologous serum (AS). First described by Fox et al. [17] and later by Tsubota et al. [18], AS drops at 20\% concentration have been shown to be an effective therapy for dry eye with Sjögren's syndrome. However, in a comparative study conducted by Tananuvat et al. [19], no significant advantage in terms of tear film stability was found in the use of AS over artificial tears. Urzua et al. [7] also did not find superiority of AS over artificial tears in the treatment of dry eye in terms of tear film break-up time (BUT), Schirmer's test or fluorescein staining. As advantages over artificial tears, AS is preservative-free and presents greater similarity to the natural healthy tear film in terms of $\mathrm{pH}$, osmolarity, and biomechanical properties [20]. This blood-derived product contains vitamins and fibronectin which have epitheliotropic effects and help to maintain ocular surface's integrity. However, AS is very poor in growth factors since platelets are eliminated in the process of its production [6]. In addition, there are many inconsistencies in AS's methods of preparation, dilution, and storage, as well as uncertainty regarding their optimal concentration.

As a new alternative, plasma rich in growth factors (PRGF) is a hemoderivative product, which is different from AS, and has been proposed for the treatment of DED [8]. An experimental study showed that PRGF exerts more potent proliferative and anti-inflammatory effects than AS on a cell culture inflammatory model [21], although there is not any clinical research in humans comparing both blood products. Similar to PRGF, autologous PRP has been proposed for the treatment of dry eye syndrome with some theoretical advantages. Autologous PRP is a preservative-free biological product from the patient's own blood and due to the presence of platelets and many biological active agents, PRP assists in the healing process. Autologous PRP has been successfully implemented in ophthalmology $[9,22]$ and can be stored at $-20^{\circ} \mathrm{C}$ for up to 3 months maintaining constant or slight variations in the concentration of the most important growth factors [23].

In the present report, we have undertaken a prospective investigation in a large scale basis to provide evidence of its effectiveness in the treatment of DED. We included 368 subjects and we show the beneficial effects of autologous PRP as monotherapy in the treatment of patients with DED. This hemoderivative product was well tolerated and almost no adverse effects (only $1.4 \%)$ were observed during the clinical study. PRP showed a considerable effect on relieving the signs and symptoms of DED. Similar results were obtained with PRGF when it was used in the treatment of evaporative dry eye, although that study included only 83 patients [24].

Regarding the 16 subjects who lost lines of vision after treatment, these were patients who suffered from pemphigoid cicatricial, GVHD, limbal stem cell deficiency (LSCD), caustication, 
or leucomas. Possibly this loss in CDVA was due to the progress of their underlying disease since most of them had improved subjective symptoms and CFS score.

Therefore, autologous PRP eye drops seems to be an attractive alternative option for the treatment of both evaporative and aqueous deficient DED, including Sjögren and nonSjögren's syndrome patients.

Significant advantages are cost effectiveness, ease of preparation and the autologous origin. The effectiveness of PRP is determined by the presence of platelets, growth factors and cytokines which are involved in proliferation, migration and differentiation of corneal epithelial cells, thus helping to maintain a proper condition of the ocular surface. Moreover, autologous PRP has been used successfully in other ocular surface disorders such as post-LASIK ocular surface syndrome, persistent epithelial defects, alkali burn, dormant ulcers, and corneal surface reconstructions in corneal perforations [25-29].

This study has some limitations regarding its design. This work is not a randomized and controlled trial. Further investigations to assess the role of this therapeutic approach in the treatment of DED versus commercial artificial tear eyedrops or other hemoderivatives are necessary to determine the best approach in the management of moderate to severe forms of this frequent ocular surface disease.

\section{CONCLUSIONS}

Monotherapy with autologous PRP eye drops has shown to be a very good option for the treatment of moderate to severe chronic DED. Significant advantages are ease of preparation, absence of preservatives, its autologous origin, safety, and minimal or no intolerance.

\section{ACKNOWLEDGEMENTS}

This study has been supported in part by a grant from European Regional Development Fund
(Fondo Europeo de Desarrollo Regional FEDER) and the Spanish Ministry of Economy and Competitiviness, Instituto Carlos III, Red Temática de Investigación Cooperativa en Salud (RETICS) "Prevención, detección precoz y tratamiento de la patología ocular prevalente, degenerativa y crónica". Subprograma "Dioptrio ocular y patologías frecuentes" (RD12/0034/ 0007). All named authors meet the International Committee of Medical Journal Editors (ICMJE) criteria for authorship for this manuscript, take responsibility for the integrity of the work as a whole, and have given final approval to the version to be published.

Disclosures. Jorge L. Alio, Alejandra E. Rodriguez, Renan Ferreira-Oliveira, Dominika Wróbel-Dudzińska and Ahmed A. Abdelghany have nothing to disclose.

Compliance with Ethics Guidelines. All procedures followed were in accordance with the ethical standards of the responsible committee on human experimentation (institutional and national) and with the Helsinki Declaration of 1964, as revised in 2013. Informed consent was obtained from all patients for being included in the study.

Open Access. This article is distributed under the terms of the Creative Commons Attribution-NonCommercial 4.0 International License (http://creativecommons.org/licenses/ by-nc/4.0/), which permits any noncommercial use, distribution, and reproduction in any medium, provided you give appropriate credit to the original author(s) and the source, provide a link to the Creative Commons license, and indicate if changes were made.

\section{REFERENCES}

1. No authors. The definition and classification of dry eyedisease: report of the definition and classification subcommittee of the international dry eye workshop. Ocul Surf. 2007;5(2):75-92. 
2. Viso E, Rodriguez-Ares MT, Gude F. Prevalence of and associated factors for dry eye in a Spanish adult population (the Salnes Eye Study). Ophthalmic Epidemiol. 2009;16(1):15-21.

3. Chia EM, Mitchell P, Rochtchina E, Lee AJ, Maroun $\mathrm{R}$, Wang JJ. Prevalence and associations of dry eye syndrome in an older population: the Blue Mountains Eye Study. Clin Exp Ophthalmol. 2003;31(3):229-32.

4. Gupta N, Prasad I, Jain R, D'Souza P. Estimating the prevalence of dry eye among Indian patients attending a tertiary ophthalmology clinic. Ann Trop Med Parasitol. 2010;104(3):247-55.

5. Alio JL, Colecha JR, Pastor S, Rodriguez A, Artola A. Symptomatic dry eye treatment with autologous platelet-rich plasma. Ophthalmic Res. 2007;39:124-9.

6. Hussain M, Shtein RM, Sugar A, Soong HK, Woodward MA, DeLoss K, Mian SI. Long-term use of autologous serum $50 \%$ eye drops for the treatment of dry eye disease. Cornea. 2014;33(12):1245-51.

7. Urzua CA, Vasquez DH, Huidobro A, Hernandez $\mathrm{H}$, Alfaro J. Randomized double-blind clinical trial of autologous serum versus artificial tears in dry eye syndrome. Curr Eye Res. 2012;37(8):684-8.

8. López-Plandolit S, Morales MC, Freire V, Grau AE, Durán JA. Efficacy of plasma rich in growth factors for the treatment of dry eye. Cornea. 2011;30(12):1312-7.

9. Alio JL, Arnalich-Montiel F, Rodriguez AE. The role of "Eye Platelet Rich Plasma" (E-PRP) for wound healing in ophthalmology. Curr Pharm Biotechnol. 2012;13(7):1257-65.

10. Tandon A, Tovey JC, Sharma A, Gupta R, Mohan RR. Role of transforming growth factor Beta in corneal function, biology and pathology. Curr Mol Med. 2010;10(6):565-78.

11. World Medical Association. World Medical Association Declaration of Helsinki: ethical principles for medical research involving human subjects. JAMA. 2013;310(20):2191-4.

12. Baudouin C, Aragona P, Van Setten G, Rolando M, Irkeç M, Benítez del Castillo J, Geerling G, Labetoulle M, Bonini S. Diagnosing the severity of dry eye: a clear and practical algorithm. ODISSEY European Consensus Group members. Br J Ophthalmol. 2014;98(9):1168-76.

13. Lemp MA, Crews LA, Bron AJ, Foulks GN, Sullivan BD. Distribution of aqueous-deficient and evaporative dry eye in a clinic-based patient cohort: a retrospective study. Cornea. 2012;31(5):472-8.
14. Bron AJ, Evans VE, Smith JA. Grading of corneal and conjunctival staining in the context of other dry eye tests. Cornea. 2003;22(7):640-50 (Review).

15. Nichols KK, Nichols JJ, Mitchell GL. The lack of association between signs and symptoms in patients with dry eye disease. Cornea. 2004;23(8):762-70.

16. Pflugfelder SC. Antiinflammatory therapy for dry eye. Am J Ophthalmol. 2004;137(2):337-42 (Review).

17. Fox RI, Chan R, Michelson JB, Belmont JB, Michelson PE. Beneficial effect of artificial tears made with autologous serum in patients with keratoconjunctivitis sicca. Arthritis Rheum. 1984;27(4):459-61.

18. Tsubota K, Goto E, Fujita H, Ono M, Inoue H, Saito I, Shimmura S. Treatment of dry eye by autologous serum application in Sjögren's syndrome. $\mathrm{Br} \mathrm{J}$ Ophthalmol. 1999;83(4):390-5.

19. Tananuvat N, Daniell M, Sullivan LJ, Yi Q, McKelvie P, McCarty DJ, et al. Controlled study of the use of autologous serum in dry eye patients. Cornea. 2001;20(8):802-6.

20. Liu L, Hartwig D, Harloff S, Herminghaus P, Wedel $\mathrm{T}$, Kasper K, et al. Corneal epitheliotrophic capacity of three different blood-derived preparations. Invest Ophthalmol Vis Sci. 2006;47(6):2438-44.

21. Anitua E, Muruzabal F, de la Fuente M, Riestra A, Merayo-Lloves J, Orive G. PRGF exerts more potent proliferative and anti-inflammatory effects than autologous serum on a cell culture inflammatory model. Exp Eye Res. 2016;151:115-21.

22. Alio JL, Rodriguez AE, WrobelDudzinska D. Eye platelet-rich plasma in the treatment of ocular surface disorders. Curr Opin Ophthalmol. $2015 ; 26(4): 325-32$.

23. Anitua E, Muruzabal F, Pino A, Merayo-Lloves J, Orive G. Biological stability of plasma rich in growth factors eye drops after storage of 3 months. Cornea. 2013;32(10):1380-6.

24. Merayo-Lloves J, Sanchez-Avila RM, Riestra AC, Anitua E, Begoña L, Orive G, Fernandez-Vega L. Safety and efficacy of autologous plasma rich in growth factors eye drops for the treatment of evaporative dry eye. Ophthalmic Res. 2016;56(2):68-73.

25. Alio JL, Rodriguez AE, Martinez LM, Rio AL. Autologous fibrin membrane combined with solid platelet-rich plasma in the management of perforated corneal ulcers: a pilot study. JAMA Ophthalmol. 2013;131(6):745-51. 
26. Alio JL, Rodriguez AE, Martinez LM. Bovine pericardium membrane (tutopatch) combined with solid platelet-rich plasma for the management of perforated corneal ulcers. Cornea. 2013;32(5):619-24.

27. Alio JL, Pastor S, Ruiz-Colecha J, Rodriguez A, Artola A. Treatment of ocular surface syndrome after LASIK with autologous platelet-rich plasma. J Refract Surg. 2007;23(6):617-9.
28. Alio JL, Abad M, Artola A, Rodriguez-Prats JL, Pastor S, Ruiz-Colecha J. Use of autologous platelet-rich plasma in the treatment of dormant corneal ulcers. Ophthalmology. 2007;114(7):1286.e1-1293.e1.

29. Ortuño-Prados VJ, Alio JL. Tratamiento de úlcera corneal neurotrófica con plasma rico en plaquetas y Tutopatch $^{\circledR}$. Arch Soc Esp Oftalmol. 2011;86(4):121-3. 